\title{
Análisis de supervivencia global y libre de enfermedad de cáncer de colon no metastásico en Bogotá (Colombia)
}

- Pedro Luis Ramos Guette

Correos electrónicos: pramos@oncocareltda,pramos152@yahoo.com

\section{Resumen}

Objetivo: evaluar la supervivencia libre de enfermedad y global de pacientes con cáncer de colon en Bogotá (Colombia).

Materiales y métodos: se trata de un estudio de cohorte bidireccional; se registró la información de las características clínicas, demográficas y los tiempos de recaída y muerte por cáncer $u$ otras causas de una cohorte de 176 pacientes con cáncer de colon durante el período 2003-2012. Los pacientes fueron seguidos en intervalos cada 4 a 6 meses, en los cuales se documentaba el estado vital y la presencia de recaídas locales o sistémicas de la enfermedad. Se realizaron análisis descriptivos y se estimaron funciones de supervivencia usando el método de Kaplan-Meier. Se efectuó análisis de regresión de Cox para identificar factores asociados a la supervivencia.
Resultados: se identificaron 176 pacientes con cáncer de colon. La mediana de seguimiento fue 31,4 meses, la edad promedio fue 62,19 años, del sexo femenino fue el $55,68 \%$. Se presentó un $14,2 \%$ de recaídas y un $8,52 \%$ de muertes, de las cuales el 93,3\% correspondió a muertes específicas por cáncer de colon. En el análisis multivariado, no se evidenció asociación de ninguno de los factores, como estadio clínico, grado histológico, tipo histológico, estado funcional y tipo de quimioterapia, con la supervivencia libre de enfermedad y global.

Conclusión: los tiempos de supervivencia libre de enfermedad y global observados en la cohorte no se relacionan en el análisis con el estadio clínico, grado histológico, tipo histológico, estado funcional o tipo de quimioterapia recibida.

Palabras clave: cáncer de colon, pronóstico, adyuvancia.

\section{Análisis de supervivencia de una cohorte con cáncer de ovario}

\section{- Pedro Luis Ramos Guette}

Correos electrónicos: pramos@oncocareltda, pramos152@yahoo.com

Resumen

Objetivo: establecer la asociación entre factores pronósticos y la supervivencia libre de enfermedad y global en una cohorte de 145 mujeres con cáncer de ovario en Bogotá (Colombia).

Materiales y métodos: se trata de un estudio de tipo cohorte bidireccional; en la clínica Oncocare se registró la información de las características clínicas, demográficas y los tiempos de recaída y muerte por cáncer u otras causas de una cohorte de 145 mujeres con cáncer de ovario durante el período 2003-2012. Las pacientes fueron seguidas en intervalos cada 4 a 6 meses, en los cuales se documentaba el estado vital y la presencia de recaídas locales o sistémicas de la enfermedad. Se realizaron análisis descriptivos y se estimaron funciones de supervivencia usando el método de Kaplan-Meier. Se llevó a cabo análisis de regresión de Cox para identificar factores asociados a la supervivencia.

Resultados: se identificaron 145 pacientes en el estudio. La mediana de seguimiento fue 23,4 meses, la edad promedio de las mujeres fue 49,6 años, el 74,5\% fue diagnosticado en estadios avanzados IC a IV, el

Palabras clave: cáncer de ovario, pronóstico, adyuvancia. tipo histológico más frecuente fue seroso. Se presentó el $24,1 \%$ de recaídas, se observó el 22,6\% de muertes por cáncer de ovario. En el análisis multivariado, se evidenció asociación de la no respuesta completa a la quimioterapia con disminución de la sobrevida libre de enfermedad y global, HR 5,86 (IC95\% 1,19-28,75) y HR 4,69 (IC95\% 1,01-22,01), respectivamente. También, la citorreducción óptima con disminución del riesgo de muerte y recaídas: HR 0,19 (IC95\% 0,07-0,54) y HR 0,39 (IC95\% 0,18-0,83), respectivamente. Los otros factores, como tipo histológico, grado histológico, estadio clínico, presencia de ascitis y estado funcional, no se relacionaron con la supervivencia libre de enfermedad y global.

Conclusión: los tiempos de supervivencia libre de enfermedad y global disminuyen si no se alcanzan respuestas completas a la quimioterapia y aumentan si hay una citorreducción óptima, pero no se asocian con ningún otro factor pronóstico analizado, y se requiere una muestra y seguimiento mayor para tener suficientes eventos que permitan conclusiones definitivas. 